\title{
Synthesis of Single-Handed Helical Spiro-Conjugated Ladder Polymers through Quantitative and Chemoselective Cyclizations
}

Wei Zheng, ${ }^{[a]}$ Tomoyuki Ikai, ${ }^{*[a]}$ and Eiji Yashima*[a]

[a] Dr. W. Zheng, Dr. T. Ikai, Prof. E. Yashima

Department of Molecular and Macromolecular Chemistry

Graduate School of Engineering, Nagoya University

Chikusa-ku, Nagoya 464-8603 (Japan)

E-mail: ikai@chembio.nagoya-u.ac.jp; yashima@chembio.nagoya-u.ac.jp

Supporting information for this article is given via a link at the end of the document.

\begin{abstract}
We report the unprecedented synthesis of one-handed helical spiro-conjugated ladder polymers with well-defined primary and secondary structures, in which the spiro-linked dibenzo[ $a, h]$ anthracene fluorophores are arranged in a one-handed twisting direction, through quantitative and chemoselective acidpromoted intramolecular cyclizations of random-coil precursor polymers composed of chiral 1,1'-spirobiindane and achiral bis[2-(4alkoxyphenyl)ethynyl]phenylene units. Intense circular dichroism (CD) and circularly polarized luminescence (CPL) were observed, whereas the precursor polymers exhibited negligible $C D$ and $C P L$ activities. The introduction of 2,6-dimethyl substituents on the 4alkoxyphenylethynyl pendants is of key importance for this simple, quantitative, and chemoselective cyclization. This strategy is applicable to the defect-free precise synthesis of other varieties of fully $\pi$-conjugated molecules and coplanar ladder polymers that have not been achieved before.
\end{abstract}

The development of a general and versatile methodology to quantitatively produce defect-free fused polycyclic aromatics and conjugated ladder-like polymers in a predictable manner has attracted significant interest because of their unique physical properties and remarkable chemical and thermal stability along with promising functions as the next-generation optoelectronic materials arising from their rigid and robust frameworks, inaccessible from traditional conjugated polymers with significant conformational freedom. ${ }^{[1]}$ Recent advances in the polymerization followed by efficient functionalization approach enable us to produce a variety of conjugated ladder polymers with specific functionalities. ${ }^{[2]}$ Among them, an acid-promoted electrophilic intramolecular multicyclization (alkyne benzannulation) developed by Goldfinger and Swager has proven to be versatile for producing a variety of ladder-type molecules and polymers, ${ }^{[3]}$ but has not always afforded completely defect-free conjugated ladder polymers because of unavoidable side reactions that occur during the electrophilic cyclization. ${ }^{[4]}$ On the basis of this acidpromoted annulative $\pi$-extension strategy, we have successfully synthesized the first defect-free one-handed helical conjugated ladder polymers and their model compounds, using a chiral bulky triptycene unit as one of the components. ${ }^{[5]}$ Apart from numerous variations in synthetic and biological helical polymers, helical conjugated ladder polymers have significant advantages over the others for their rigid three-dimensional helical geometry with a strictly limited conformational freedom leading to development of electronic and photonic devices for sensing and detection of
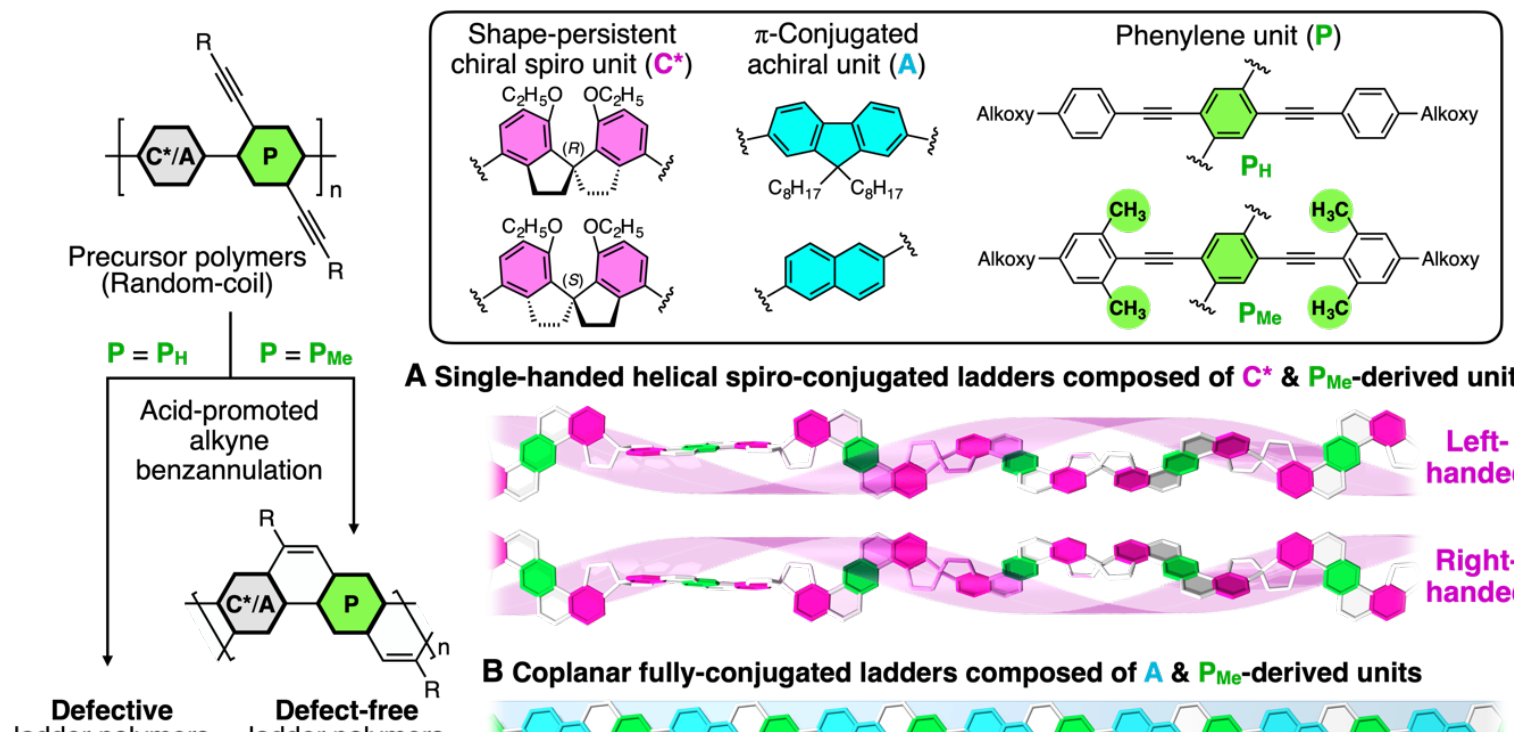

A Single-handed helical spiro-conjugated ladders composed of $\mathrm{C}^{*} \& \mathrm{P}_{\mathrm{Me}}$-derived units

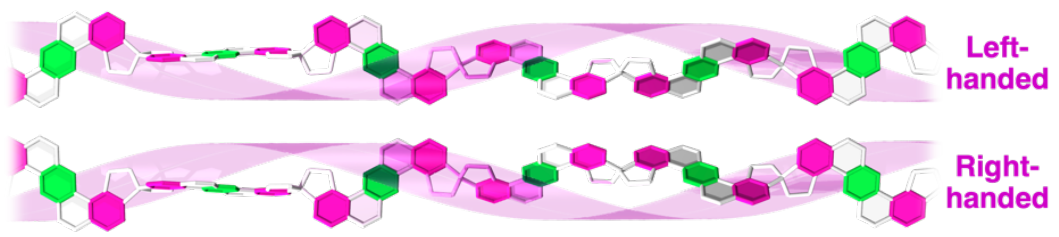

B Coplanar fully-conjugated ladders composed of $\mathrm{A} \& \mathrm{P}_{\mathrm{Me}}$-derived units ladder polymers ladder polymers

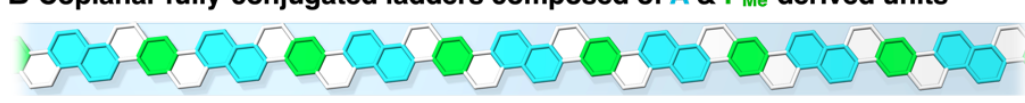

Figure 1. Schematic illustration of quantitative and chemoselective acid-promoted alkyne benzannulations assisted by introducing the 2,6-dimethyl substituents on the 4-alkoxyphenyl pendants to produce defect-free single-handed helical spiro-conjugated (A) and coplanar fully-conjugated (B) ladder polymers. 

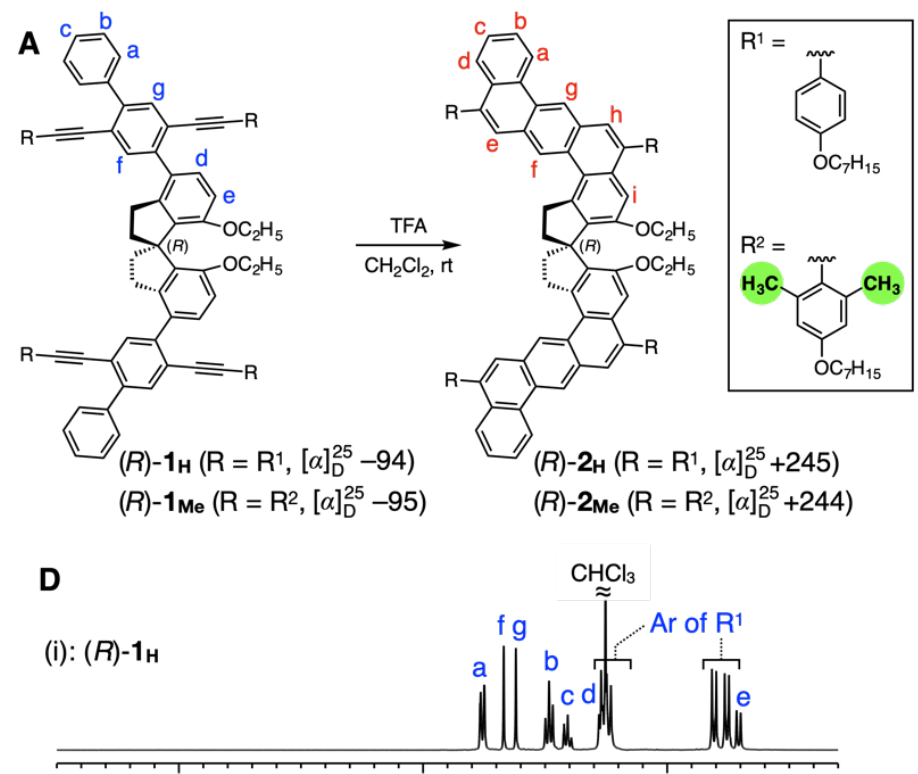

(ii): As-synthesized product mixture $\left((R)-\mathbf{2}_{\mathrm{H}}\right.$ and byproduct $\left.((R)-2 \mathrm{H}-\mathrm{b})\right)$

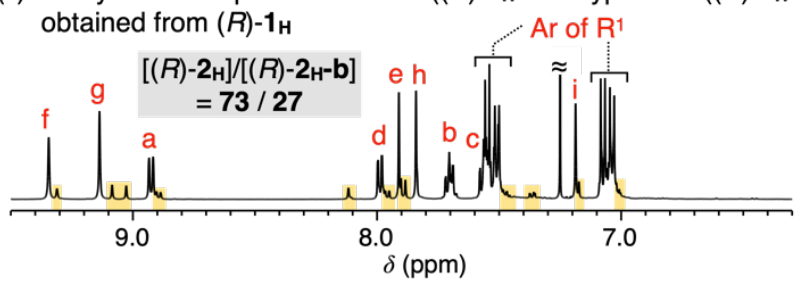

B

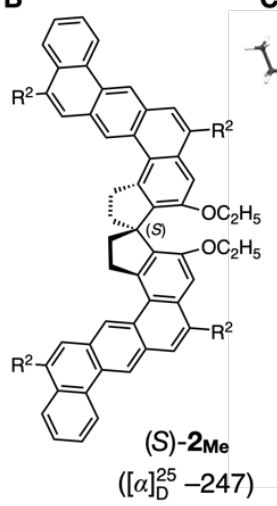

C

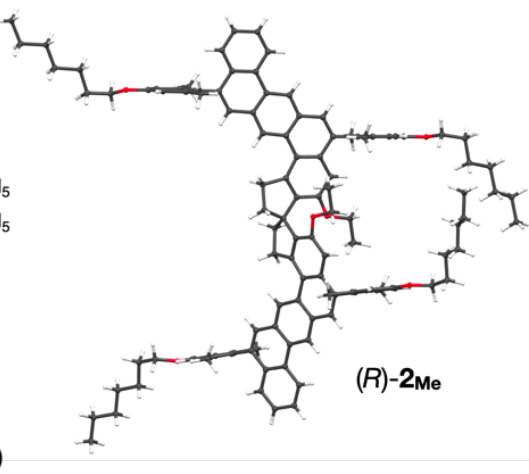

(iii): (R)-1 $\mathbf{1}_{\text {Me }}$

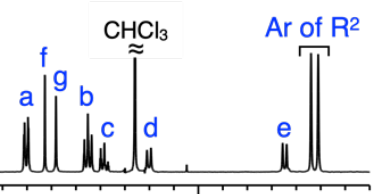

(iv): As-synthesized product $((R)-\mathbf{2 M e})$ obtained from $(R) \mathbf{1}_{\text {Me }}$

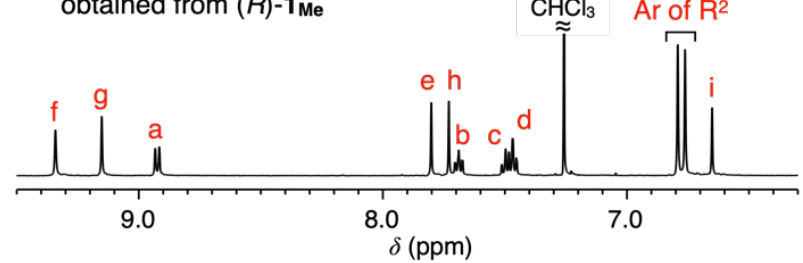

Figure 2. (A) Synthesis of optically-active spiro-conjugated ladder-type molecules $((R)-2)$ through acid-promoted alkyne benzannulations of $(R)-1$. (B) Structure of $(S)-2_{\mathrm{Me}}$ (C) X-ray crystal structure of $(R)$-2. Thermal ellipsoids are represented at the $50 \%$ probability level. (D) ${ }^{1} \mathrm{H} \mathrm{NMR} \mathrm{spectra}\left(500 \mathrm{MHz}, \mathrm{CDCl} 3,25{ }^{\circ} \mathrm{C}\right)$ of $(R)$-1 $\mathrm{H}_{\mathrm{H}}$ (i), $(R)-1_{\mathrm{Me}}(\mathrm{iii})$, and as-synthesized cyclization products obtained from $(R)-\mathbf{1}_{\mathrm{H}}$ (ii) and $(R)-\mathbf{1}_{\mathrm{Me}}(\mathrm{iv})$. For the signal assignments and the corresponding IR spectra, see Figures S1-S4 and S5A-D, respectively. Yellow colors in (ii) denote the aromatic protons from the byproduct, $(R)-2 \mathrm{H}-\mathrm{b}$ (see Figures $\mathrm{S} 6$ and $\mathrm{S} 7$ ).

chirality of chiral molecules ${ }^{[6]}$ as well as chiral materials for separating enantiomers as chiral packing materials. ${ }^{[5 c]}$

Spiro-conjugated frameworks have been utilized as attractive and useful building blocks for optically-active spirobridged ladder-type oligomers ${ }^{[7]}$ due to the axially chiral spiro scaffold, thereby forming unique orthogonally-conjugated chiral architecture with intriguing circularly polarized luminescence (CPL). Chiral spirobifluorene units were also employed to construct optically-active helical ladder-like polymers by Takata and co-workers, ${ }^{[8]}$ but these structures were not fully characterized.

We now report the first example of single-handed helical spiro-conjugated ladder polymers that can be readily synthesized from random-coil precursor polymers containing optically-active 1,1 '-spirobiindane units as a spiro-type chiral linkage in the main chain (Figure 1A). During the course of our studies, we found that introduction of 2,6-dimethyl substituents on the 4-alkoxyphenyl pendants of the precursor polymers enabled complete chemoselective intramolecular cyclizations during acid-promoted alkyne benzannulations by suppressing undesired reaction pathways. Left- and right-handed helical spiro-conjugated ladder polymers with well-defined primary and secondary structures were thus quantitatively produced, showing characteristic circular dichroism (CD) and CPL that were largely different from those of the precursor polymers. We also show that this simple, quantitative, and chemoselective cyclization approach, assisted by introducing the 2,6-dimethyl substituents on the 4alkoxyphenyl pendants, can be applicable to the defect-free precise synthesis of other polycyclic aromatics and $\pi$-conjugated ladder polymers composed of fluorene and naphthalene moieties (Figure 1B).

We first examined the feasibility of an acid-promoted intramolecular alkyne benzannulation using trifluoroacetic acid (TFA) to a chiral 1,1'-spirobiindane-based model unimer, $(R)-1 \mathrm{H}$, containing two bis[2-(4-alkoxyphenyl)ethynyl]phenylene pendants (Figure $2 \mathrm{~A}$ ). However, the reaction of $(R)-\mathbf{1}_{\mathrm{H}}$ with TFA afforded a mixture of the anticipated $(R)-2 \mathrm{H}$ and a byproduct, $(R)$ $2 \mathrm{H}-\mathbf{b}$, at a ratio of $73: 27$, as suggested by the ${ }^{1} \mathrm{H}$ NMR spectrum of the product mixture (Figures $2 \mathrm{D}(\mathrm{i}, \mathrm{ii})$ and $\mathrm{S} 6$ ); $(R)-\mathbf{2} \mathrm{H}-\mathrm{b}$ was produced through an electrophile-induced intramolecular cyclization accompanied by rearrangements of the aryl pendants. ${ }^{[9,10]}$ During the course of our studies, we found that the introduction of 2,6-dimethyl substituents on the 4-alkoxyphenyl pendants of the cyclization precursor, namely, $(R)-1$ me (Figure $2 \mathrm{~A}$ ), allowed the acid-promoted cyclization to proceed perfectly, without any side reactions, thus quantitatively and chemoselectively producing $(R)-2 \mathrm{Me}$ as a single product, as supported by its simple ${ }^{1} \mathrm{H}$ NMR spectrum (Figure $2 \mathrm{D}$ (iii,iv)). ${ }^{[11]}$ The structure of $\mathbf{2}_{\mathrm{Me}}$ was unambiguously determined by singlecrystal X-ray crystallography (Figure 2C). ${ }^{[12]}$

Next, we applied this quantitative and chemoselective cyclization to the synthesis of one-handed helical spiroconjugated ladder polymers with controlled primary and secondary structures. Poly-5 $\mathbf{R}_{\mathrm{Me}}$, composed of alternating chiral 1,1'-spirobiindane and achiral bis[2-(4-alkoxy-2,6dimethylphenyl)ethynyl]phenylene segments, was synthesized by the copolymerization of $(R)-\mathbf{3}$ with $\mathbf{4}_{\mathrm{Me}}$ via Suzuki-Miyaura coupling (Figure $3 \mathrm{~A})$. The number-average molar mass $\left(M_{\mathrm{n}}\right)$ of the 
A<smiles>CCc1c(B2OC(C)(C)C(C)(C)O2)ccc(OC)c1[C@@]12CC[C@H]1c1c(B3OC(C)(C)C(C)(C)O3)ccc(OC)c12</smiles><smiles>[R]C#Cc1cc(Br)c(C#C[14CH3])cc1Br</smiles>

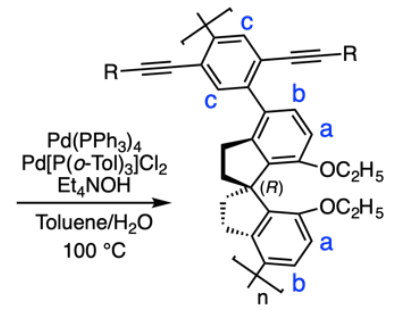

poly-5R $\mathbf{R}_{\mathrm{Me}}\left([\alpha]_{D}^{25}-107\right)$

$M_{\mathrm{n}}: 1.16 \times 10^{4}$

$M_{\mathrm{w}} / M_{\mathrm{n}}: 1.22$

B (i): poly-5R
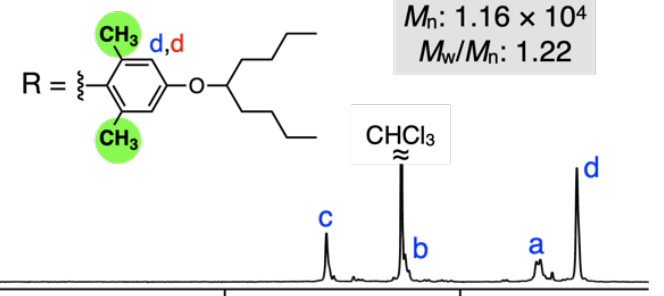

(ii): As-synthesized product (poly-6 $\mathbf{R}_{\mathrm{Me}}$ ) obtained from poly-5 $\mathbf{R}_{\mathrm{Me}}$

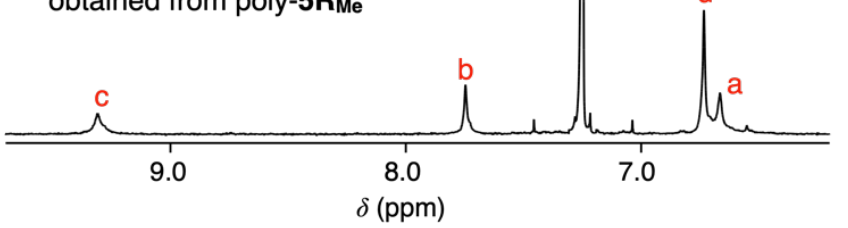

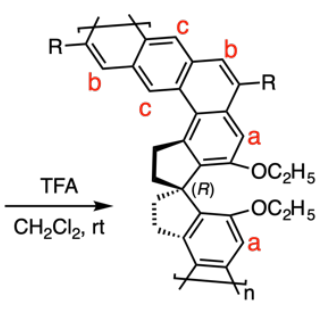

poly- $\left.6 \mathbf{R}_{\mathrm{Me}}\left([\alpha]_{D}^{25}+298\right)\right)$

D
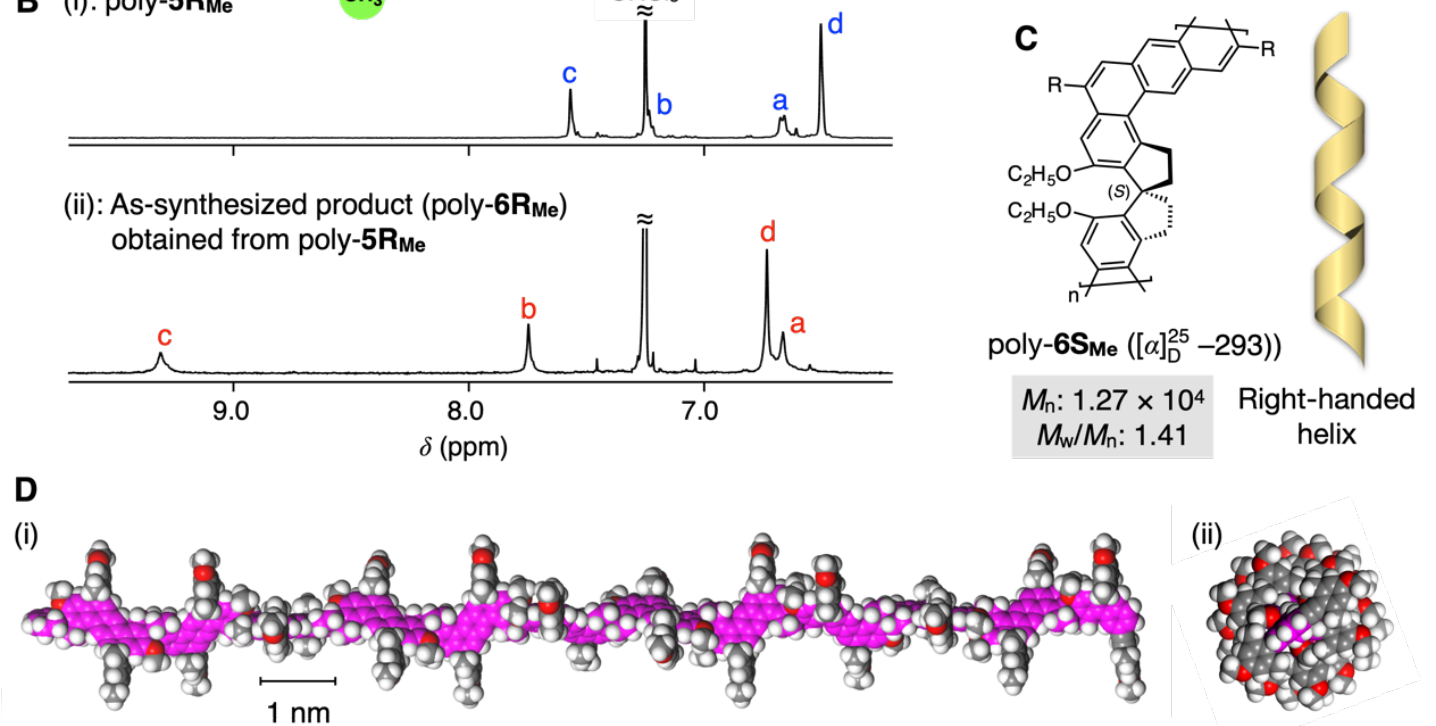

$M_{\mathrm{n}}: 1.20 \times 10^{4}$ Left-handed

$M_{\mathrm{w}} / M_{\mathrm{n}}: 1.32 \quad$ helix

(ii)

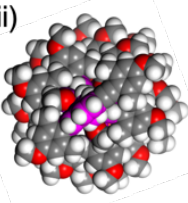

Figure 3. (A) Synthesis of left-handed helical spiro-conjugated ladder polymer (poly-6 $\mathbf{R}_{\mathrm{Me}}$ ) through Suzuki-Miyaura coupling copolymerization of $(R)-3$ and $\mathbf{4}_{\mathrm{Me}}$,

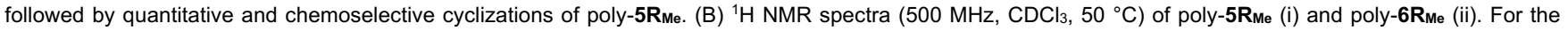
signal assignments, see Figures S8 and S9. (C) Structure of right-handed helical poly-6S $\mathrm{Me}$. (D) Side (i) and top (ii) views of a left-handed helical poly-6RMe structure with 12 repeating units optimized by molecular mechanics (MM) calculations (MMFF94s force field). For simplicity, all alkoxy pendants are replaced with methoxy groups. The carbon atoms of the helical spiro-conjugated ladder backbone are highlighted in purple.

resulting poly- $5 \mathbf{R}_{\mathrm{Me}}$ was estimated to be $1.2 \times 10^{4}$ by sizeexclusion chromatography (SEC), which corresponds to a degree of polymerization (DP) of ca. 13. Subsequently, poly-5RMe was treated with TFA to transform its random-coil conformation to a rigid-rod one-handed helical ladder structure (Figure 3A). After reaction for $12 \mathrm{~h}$, the ethynyl groups in poly- $5 \mathrm{R}_{\mathrm{Me}}$ were completely consumed, as confirmed by IR analysis (Figure S5I,J). The $M \mathrm{n}$ and molar-mass dispersity $\left(M_{\mathrm{w}} / M_{\mathrm{n}}\right)$ of poly-6RMe $\left(M_{\mathrm{n}}=1.2 \times 10^{4}\right.$, $\left.M_{\mathrm{w}} / M_{\mathrm{n}}=1.3\right)$ remained virtually unchanged after cyclization, indicating that undesired intermolecular electrophilic aromatic additions, which would cause an irregular cross-linked structure, did not occur. The matrix-assisted laser desorption-ionization time-of-flight mass spectrum of poly- $6 \mathbf{R}_{\mathrm{Me}}$ showed main series of peaks over a region of more than $1.0 \times 10^{4} \mathrm{~m} / \mathrm{z}$ with a regular interval of approximately $923.3(\mathrm{~m} / \mathrm{z})$ mass, corresponding to the molar mass of the repeating unit (Figure S10). The simple ${ }^{1} \mathrm{H}$ NMR spectrum of poly-6 $\mathbf{R}_{\text {Me }}$ (Figure $3 \mathrm{~B}(\mathrm{ii})$ ), in which all the proton resonances could be unequivocally assigned by $2 \mathrm{D} N M R$ analysis (Figure S9), supports its regular ladder structure. On the other hand, when poly-5 $\mathbf{R}_{\mathbf{H}}$, which lacks the 2,6-dimethyl substituents, was treated with TFA, a spiro-conjugated ladder-like polymer (poly-6 $\mathbf{R}_{\mathbf{H}}$ ) but with an irregular backbone structure probably composed of two different repeating units was produced as shown in its complicated ${ }^{1} \mathrm{H}$ NMR spectrum (Figure S11), which is in good agreement with the results obtained for the model unimer precursors, $\mathbf{1}_{\mathrm{H}}$ and $\mathbf{1}_{\mathrm{Me}}$ (Figure 2).

Figure 3D shows a possible helical ladder structure of poly$\mathbf{6 R}_{\mathrm{Me}}$ with 12 repeating units optimized by molecular mechanics calculations. The axially chiral $(R)$-spiro repeating linkages with the limited conformational freedom inevitably force the formation of orthogonally-conjugated dibenzo[a,h]anthracene units in the entire ladder polymer backbone in a counterclockwise twisting manner, thus producing a rigid-rod left-handed helical ladder structure. Obviously, when poly-5 $\mathbf{S}_{\mathrm{Me}}$ was allowed to react with TFA, a defect-free ladder polymer, poly- $6 S_{\mathrm{Me}}$, with an opposite right-handed helical structure was quantitatively formed (Figure 3C). Rigid-rod one-handed helical structures of the spiroconjugated ladder polymers were further supported by their chiroptical properties. After cyclization, the optical rotation of the ladder polymer (poly-6 $\mathbf{R}_{\mathrm{Me}}$ ) showed a large positive value $\left([\alpha]_{\mathrm{D}}{ }^{25}\right.$ $+298^{\circ}$ ), the sign of which was opposite to that of the precursor polymer, poly-5RMe $\left([\alpha]_{D}{ }^{25}-107^{\circ}\right)$ (Figure $\left.3 A\right)$. Moreover, the helical poly-6RMe and poly-6S $\mathrm{S}_{\mathrm{Me}}$ exhibited intense split-type Cotton effects $\left(\left|\Delta \varepsilon_{\max }\right|=318\right)$ with mirror images to each other in the absorption regions of the dibenzo[a,h]anthracene units $(250$ to $400 \mathrm{~nm}$ ), while the precursor polymers (poly-5 $\mathbf{R}_{\mathrm{Me}}$ and poly$\mathbf{5} \mathbf{S}_{\text {Me }}$ ) showed negligibly weak CD bands $(|\Delta \varepsilon \max |=11.4)$ (Figure 4A). A similar ladderization-induced dramatic CD enhancement 

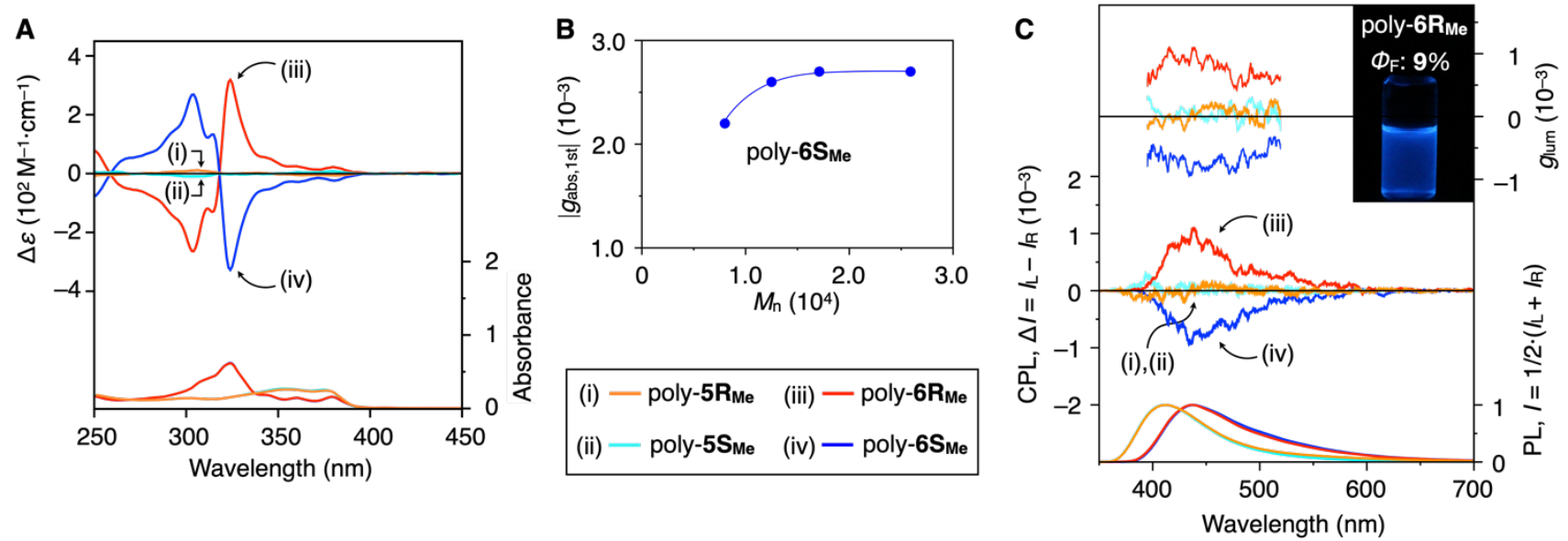

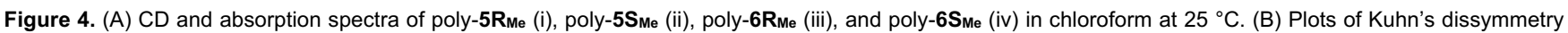
factors at the first Cotton effect $\left(\mid g_{\mathrm{abs}}\right.$ 1st $\left.\mid\right)$ of poly-6S $\mathbf{S}_{\mathrm{Me}}$ versus the $M_{\mathrm{n}}$ value. For the SEC fractionation and the corresponding CD spectra, see Figure S14. (C)

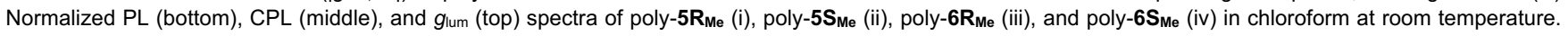
The $g_{\text {lum }}$ values are defined as $2\left(I_{L}-I_{R}\right) /\left(I_{L}+I_{R}\right)$, where $I_{L}$ and $I_{R}$ are the PL intensities of the left- and right-handed circularly polarized light, respectively. $\lambda_{\text {ex }}=300$ $\mathrm{nm}$. Inset: Photograph of poly- $6 \mathbf{R}_{\mathrm{Me}}$ in chloroform under irradiation at $365 \mathrm{~nm}$. A fluorescence quantum yield $\left(\Phi_{\mathrm{F}}\right)$ is also shown. [Polymer] $=5.0 \times 10^{-5} \mathrm{M}$ (calculated based on the monomer units).

accompanied by a significant change in the optical rotation was also observed for the model $(R)-2 \mathrm{Me}$ and $(S)-2 \mathrm{Me}$ (Figures $2 \mathrm{~A}, \mathrm{~B}$ and S12A). ${ }^{[13]}$ The exciton-coupled CD spectral patterns observed for poly-6Rme and poly-6S $S_{\text {Me }}(\text { Figure } 4 A)^{[14]}$ as well as those for their model compounds $(R)-2 \mathrm{Me}$ and $(S)-2 \mathrm{Me}$ (Figure S12A) suggest the spiro-linked dibenzo[a,h]anthracene chromophore

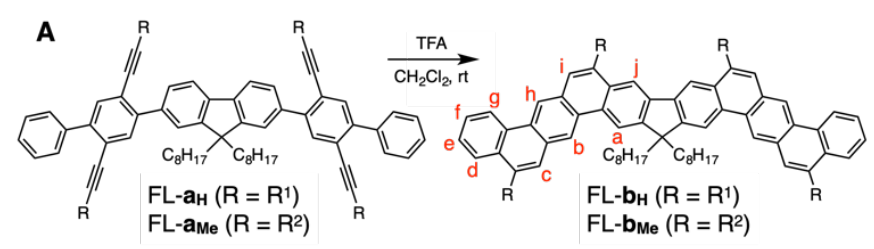

units are arranged in right- and left-handed twisting direction, respectively. This assignment is consistent with the crystal structure of $(R)-2 \mathrm{Me}$ (Figure $2 \mathrm{C}$ ). Interestingly, the CD intensity at the first Cotton effect (Kuhn's dissymmetry factor $\left.\left(\left|g_{\mathrm{abs}, 1 \mathrm{st}}\right|\right)\right)$ of poly-6S $S_{M e}$ tended to increase with an increase in the $M_{\mathrm{n}}$ and reached an almost maximum value at an $M_{\mathrm{n}}$ of ca. $1.7 \times 10^{4}$
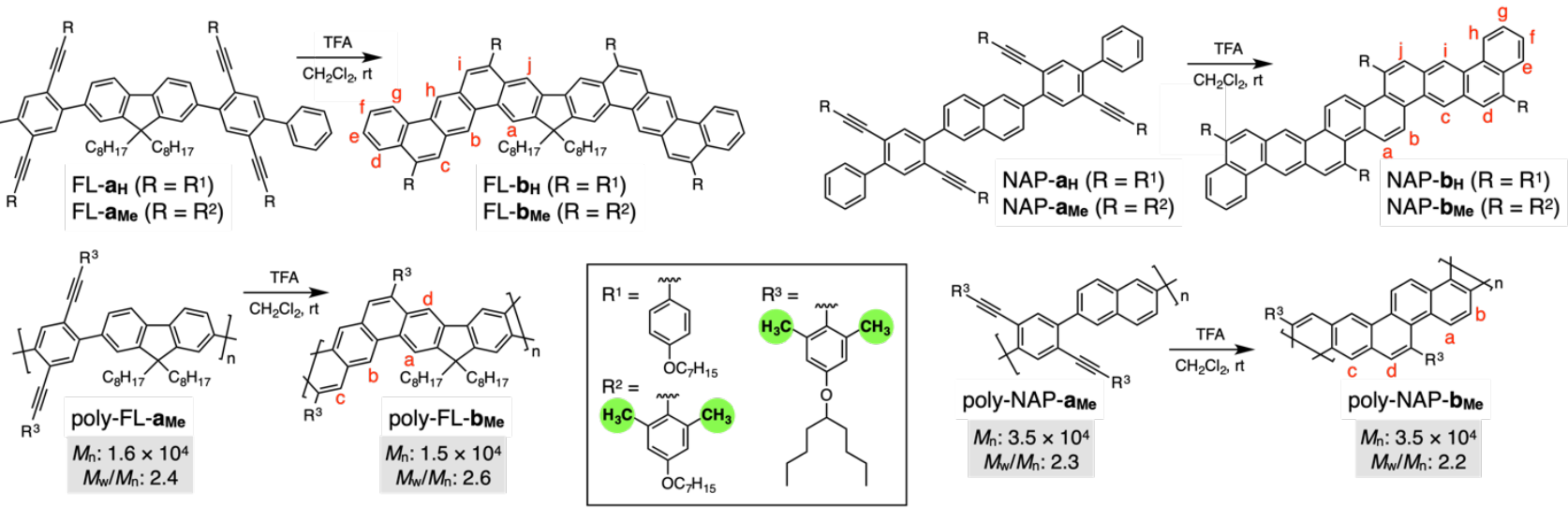

B

(i): As-synthesized product mixture obtained from FL-aн

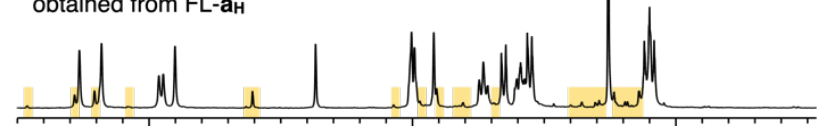

(iii): As-synthesized product (FL-b $\mathrm{b}_{\mathrm{Me}}$ ) obtained from FL-ame

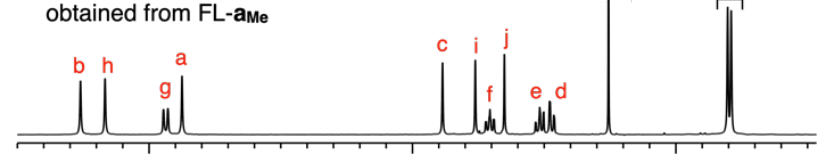

(v): As-synthesized product (poly-FL-b $\mathrm{b}_{\mathrm{Me}}$ ) obtained from poly-FL-ame

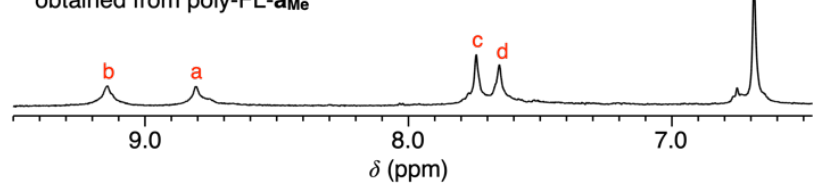

(ii): As-synthesized product mixture obtained from NAP-aH

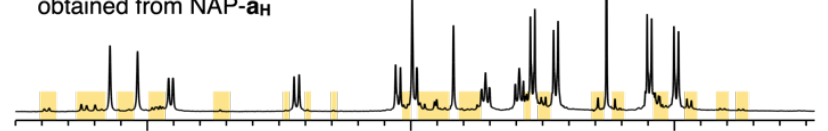

(iv): As-synthesized product (NAP-bMe) obtained from NAP-ame

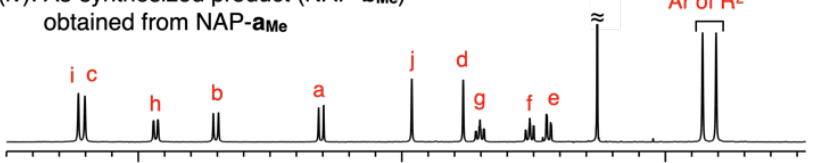

(vi): As-synthesized product (poly-NAP-b $\mathbf{b}_{\mathrm{Me}}$ ) obtained from poly-NAP-ame

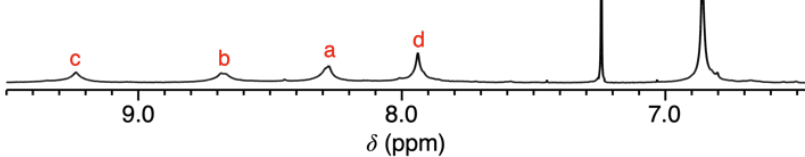

Figure 5. (A) Synthesis of fully conjugated ladder-type molecules (FL-b and NAP-b) and polymers (poly-FL-b $\mathbf{b}_{\mathrm{Me}}$ and poly-NAP-b $\mathbf{b}_{\mathrm{Me}}$ ) through acid-promoted alkyne

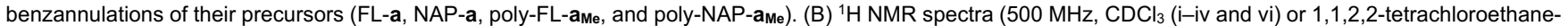
$d_{2}(\mathrm{v}), 25^{\circ} \mathrm{C}(\mathrm{i}-\mathrm{iv}), 80^{\circ} \mathrm{C}(\mathrm{v})$, or $50^{\circ} \mathrm{C}(\mathrm{vi})$ ) of as-synthesized cyclization products obtained from the precursors, FL-aн (i), NAP-aн (ii), FL-ame (iii), NAP-ame (iv), polyFL-ame (v), and poly-NAP-ame (vi). For the signal assignments in (iii)-(vi), see Figures S16-S19, respectively. ${ }^{1} \mathrm{H}$ NMR spectra of the corresponding cyclization precursors are shown in Figure S20. Yellow colors in (i) and (ii) denote the aromatic protons from byproducts. 
(corresponding to approximately 19 repeating units) (Figure 4B); its $g_{\mathrm{abs}, 1 \text { st }}$ value $\left(2.7 \times 10^{-3}\right)$ was approximately twice that of the unimer model, $(S)-2 \mathrm{Me}\left(\left|g_{\mathrm{abs}, 1 \mathrm{st}}\right|=1.6 \times 10^{-3}\right)$ (Figure S14C). The left- and right-handed helical poly-6RMe and poly- $6 S_{M e}$ also exhibited clear CPL signals with opposite signs in the corresponding fluorescence regions of the spiro $\pi$-conjugated backbones (Figure 4C). ${ }^{[15]}$ In contrast, the precursor (poly-5 $\mathbf{R}_{\mathrm{Me}}$ and poly-5 $\mathbf{S}_{\mathrm{Me}}$ ) and defective (poly-6 $\mathbf{R}_{\mathrm{H}}$ ) polymers produced almost no or very weak CPL signals, respectively (Figures $4 \mathrm{C}$ and $\mathrm{S} 15)$. These results reveal a crucial role of the defect-free onehanded helical spiro-conjugated ladder structure in their outstanding chiroptical properties.

Fluorene- (FL-b $b_{M e}$ and poly-FL- $b_{M e}$ ) and naphthalene(NAP-bme and poly-NAP-bme) based $\pi$-conjugated molecules and polymers with exquisite ladder structures could also be readily synthesized from the corresponding cyclization precursors through the quantitative and chemoselective cyclization approach only when the two methyl substituents were introduced at the 2,6positions on the 4-alkoxyphenyl pendants (Figure $5 \mathrm{~A}$ and $\mathrm{B}$ (iiivi)). ${ }^{[16]}$ Again, the acid-promoted alkyne benzannulations of FL-aH and NAP-a $\mathbf{a}_{\mathbf{H}}$ without 2,6-dimethyl substituents afforded product mixtures (Figure $5 \mathrm{~B}(\mathrm{i}, \mathrm{ii})$ ).

In summary, both right- and left-handed helical spiroconjugated ladder polymers with well-defined primary and secondary structures, showing noticeable CD and CPL activities, were successfully synthesized, for the first time, by taking advantage of the versatile acid-promoted alkyne benzannulations combined with the rational design of precursor polymers for constructing an exquisite helical architecture arising from the unique axially chiral spiro skeleton. The present simple, quantitative, and chemoselective cyclization relies on the introduction of 2,6-dimethyl substituents to the 4alkoxyphenylethynyl pendants of the precursor polymers. We believe that the present methodology can be further applied to the synthesis of not only a novel family of spiro-conjugated ladder polymers with a one-handed helical geometry but also a wide variety of other fully-conjugated ladder-type molecules and polymers with unique helical, coplanar, and zig-zag geometries through an appropriate design of chiral and/or achiral monomeric units, thus providing a promising class of advanced materials for organic electronics/spintronics, gas transport/storage, and (chiral) separations. ${ }^{[1,5 c, 17]}$ Work towards these goals is currently in progress and will be reported in due course.

\section{Acknowledgements}

We thank Professor Makoto Yamashita and Dr. Jun-ichi Ito (Nagoya University) for X-ray crystallographic analysis of $\mathbf{2}$ Me, Dr. Keiko Kuwata (Nagoya University) for high-resolution mass spectrometric analysis, Dr. Nozomu Suzuki (Nagoya University) for molecular modeling, and Mr. Kokoro Takayama for CPL spectral measurements. This work was supported in part by JSPS KAKENHI (Grant-in-Aid for Specially Promoted Research, No. $18 \mathrm{H} 05209$ (E.Y. and T.I.)).

\section{Conflict of interest}

The authors declare no conflict of interest.
Keywords: alkyne benzannulations $\cdot$ ladder polymer $\cdot$ helical structure $\cdot$ spiro-conjugated structures $\cdot$ circularly polarized luminescence

[1] For recent reviews, see: a) Z. X. Cai, M. A. Awais, N. Zhang, L. P. Yu, Chem 2018, 4, 2538-2570; b) J. H. Chen, K. Yang, X. Zhou, X. G. Guo, Chem. - Asian J. 2018, 13, 2587-2600; c) W. Chen, F. Yu, Q. Xu, G. Zhou, Q. Zhang, Adv. Sci. 2020, 7, 1903766; d) Z.-D. Yu, Y. Lu, J.-Y. Wang, J. Pei, Chem. - Eur. J. 2020, 26, 16194-16205

[2] For recent reviews, see: a) W. Chalifoux, W. Yang, Synlett 2017, 28, 625-632; b) Y. C. Teo, H. W. H. Lai, Y. Xia, Chem. - Eur. J. 2017, 23 14101-14112; c) X. Y. Wang, A. Narita, K. Müllen, Nat. Rev. Chem. 2018, 2, 0100; d) C. Z. Zhu, A. J. Kalin, L. Fang, Acc. Chem. Res. 2019, 52, 1089-1100; e) A. Jolly, D. D. Miao, M. Daigle, J. F. Morin, Angew. Chem., Int. Ed. 2020, 59, 4624-4633.

[3] a) M. B. Goldfinger, T. M. Swager, J. Am. Chem. Soc. 1994, 116, 78957896; b) M. B. Goldfinger, K. B. Crawford, T. M. Swager, J. Am. Chem. Soc. 1997, 119, 4578-4593.

[4] M. B. Goldfinger, K. B. Crawford, T. M. Swager, J. Org. Chem. 1998, 63, 1676-1686.

[5] For optically-active helical ladder polymers, see: a) Y. Dai, T. J. Katz, D. A. Nichols, Angew. Chem., Int. Ed. Engl. 1996, 35, 2109-2111; b) T. Iwasaki, Y. Kohinata, H. Nishide, Org. Lett. 2005, 7, 755-758; c) T. Ikai, T. Yoshida, K.-i. Shinohara, T. Taniguchi, Y. Wada, T. M. Swager, J. Am. Chem. Soc. 2019, 141, 4696-4703.

[6] E. Yashima, N. Ousaka, D. Taura, K. Shimomura, T. Ikai, K. Maeda, Chem. Rev. 2016, 116, 13752-13990.

[7] S. H. Liu, D. B. Xia, M. Baumgarten, Chempluschem 2021, DOI: 10.1002/cplu.202000467.

[8] Z. Yi, H. Okuda, Y. Koyama, R. Seto, S. Uchida, H. Sogawa, S. Kuwata, T. Takata, Chem. Commun. 2015, 51, 10423-10426.

[9] T. Ikai, S. Yamakawa, N. Suzuki, E. Yashima, Chem. - Asian J. 2021, DOI: 10.1002/asia.202100035.

[10] The structure of $(R)-\mathbf{2} \mathrm{H}-\mathrm{b}$ was elucidated by 2D NMR after isolation (Figure S7).

[11] A similar effect of the 2,6-dimethyl substituents on the 4alkoxyphenylethynyl moieties on the synthesis of extended fused-ring quinquephenyl oligomers was reported by Swager and coworkers.

[12] Racemic crystals of ( $R$ and $S)-\mathbf{2}_{\mathrm{Me}}$ were obtained by slow evaporation of its solution in chloroform/methanol (see Section 4 in the Supporting Information (SI)). Only the structure of one of the enantiomers is shown in Figure 2C.

[13] Poly-6RMe and (R)-2me showed no concentration-, temperature-, and solvent-dependent $C D$ spectral changes (Figure S13), therefore, the formation of aggregates could be excluded.

[14] Harada, N.; Nakanishi, K. Circular Dichroic Spectroscopy: Exciton Coupling in Organic Stereochemistry; University Science Book: Mill Valley, CA, 1983.

[15] For leading examples of CPL-active spiro compounds, see: a) M. Murai, Y. Takeuchi, K. Yamauchi, Y. Kuninobu, K. Takai, Chem. - Eur. J. 2016, 22, 6048-6058; b) K. Takase, K. Noguchi, K. Nakano, Org. Lett. 2017, 19, 5082-5085; c) J. Oniki, T. Moriuchi, K. Kamochi, M. Tobisu, T. Amaya, J. Am. Chem. Soc. 2019, 141, 18238-18245; d) S. Y. Yang, Y. K. Wang, C. C. Peng, Z. G. Wu, S. Yuan, Y. J. Yu, H. Li, T. T. Wang, H. C. Li, Y. X. Zheng, Z. Q. Jiang, L. S. Liao, J. Am. Chem. Soc. 2020, 142, 17756-17765.

[16] Scherf and co-workers reported an acid-promoted alkyne benzannulation of a similar fluorene-based precursor polymer without 2,6-dimethyl substituents on the 4-alkoxyphenyl pendants, resulting in a ladder polymer with an irregular backbone structure composed of regioisomeric syn- and anti-repeating units, see: F. Trilling, M.-K. Ausländer, U. Scherf, Macromolecules 2019, 52, 3115-3122.

[17] Z. X. Low, P. M. Budd, N. B. McKeown, D. A. Patterson, Chem. Rev. 2018, 118, 5871-5911. 